\title{
No Effect of an Automated Bolus Calculator in Pediatric Patients with Type 1 Diabetes on Multiple Daily Injections: The Expert Kids Study
}

\author{
Jens Otto Broby Madsen, MD, Kristina Casteels, MD, PhD, ${ }^{2,3}$ \\ Steffen Fieuws, $\mathrm{PhD}^{4}$ Kurt Kristensen, $\mathrm{PhD}^{5}$ Koen Vanbrabant, $\mathrm{PhD}^{4}$ \\ Marta Ramon-Krauel, MD, $\mathrm{PhD}^{6}$ and Jesper Johannesen, $\mathrm{MD}, \mathrm{DMSC}^{1,7}$; on behalf \\ of the $A B C$ consortium*
}

\begin{abstract}
Background: This multicenter crossover study investigated the potential beneficial effect of an automated bolus calculator $(\mathrm{ABC})$ in children and adolescents with type 1 diabetes (T1D) treated with multiple daily injections (MDI).

Methods: Participants were randomized to either begin or end with a 5 months intervention versus their regular treatment regimen (control), separated by a 2 months washout period. During the intervention participants were carefully instructed to use the ABC (Accu-Check Aviva Expert) versus manual insulin calculations during the control period. Participants between 8 and 18 years of age with T1D were recruited from clinics in Denmark, Belgium, and Spain. Inclusion criteria included T1D for $>1$ year, a minimum of 3 months MDI treatment before inclusion, and $\mathrm{HbA} 1 \mathrm{c}$ of $7.5 \%-11 \%$ (57-97 mmol/mol). Improvement in $\mathrm{HbA} 1 \mathrm{c}$ was the main outcome, and improved quality of life (QoL) and glucose variability (time spent in target glucose) were secondary outcomes. Results: A total of 65 patients with a mean age of 13.25 years and a mean $\mathrm{HbA} 1 \mathrm{c}$ of $8.25 \%(66.7 \mathrm{mmol} / \mathrm{mol})$ were included. Midway evaluation after 2 months of intervention showed no significant difference from the standard care $(0.297,95 \%$ confidence interval $[\mathrm{CI}]:-0.645$ to $0.054 ; P=0.10)$. The difference remained insignificant after the 5 months of intervention $(-0.143$ [95\% CI: -0.558 to $0.272 ; P=0.51])$. Using the $\mathrm{ABC}$ did not change the time spent in target glucose range, nor did it change the QoL.

Conclusions: Our study did not demonstrate beneficial additive effects of an $\mathrm{ABC}$ in children and adolescents with T1D treated with MDI neither in HbA1c, nor in any other endpoint investigated.
\end{abstract}

Keywords: Automated bolus calculator, type 1 diabetes, Pediatric, Multiple daily injections.

Introduction

B LOOD GLUCOSE LEVELS close to the normal range are essential for the short- and long-term well-being of children and adolescents with type 1 diabetes (T1D). ${ }^{1}$
Intensive insulin treatment, in the form of multiple daily injections (MDI), has been proven to both delay onset and slow progression of known long-term diabetic complications. ${ }^{2}$ However, this form of treatment can be challenging for many children and adolescents as it involves several daily

${ }^{1}$ Department of Pediatrics, Herlev University Hospital, Herlev, Denmark.

${ }^{2}$ Department of Pediatrics, University Hospitals Leuven, Leuven, Belgium.

${ }^{3}$ Department of Development and Regeneration, University of Leuven, Leuven, Belgium.

${ }_{4}^{4}$ Interuniversity Institute for Biostatistics and Statistical Bioinformatics, KU Leuven-University of Leuven \& Universiteit Hasselt, Leuven, Belgium.

${ }^{5}$ Department of Pediatrics, Skejby University Hospital, Aarhus, Denmark.

${ }^{6}$ Department of Endocrinology, Institut de Recerca Sant Joan de Deu, Hospital Sant Joan de Deu, Barcelona, Spain.

${ }^{7}$ Faculty of Health and Medical Sciences, University of Copenhagen, Copenhagen, Denmark.

*See Acknowledgments section. 
self-monitored blood glucose (SMBG) measurements and numerous repeated insulin administrations.

Rational insulin therapy should ensure flexibility in the quantity, quality, and choice of foods without jeopardizing postprandial glycemic control (blood glucose levels after a meal). To achieve this, the preprandial (before meal) bolus must be calculated carefully based on the prevailing glucose level and the estimated carbohydrate content of the meal (carb-counting). Carb-counting is a meal planning approach for patients with T1D focusing on carbohydrate as the primary nutrient affecting postprandial glycemic response.

Every day several different factors influence the blood glucose. Besides insulin dosing, these factors include eating habits, physical activity, and both emotional and physical stress. All of the aforementioned factors make manual calculation of the best fitting insulin dose for a given meal, in a given situation, a challenging and time-consuming task.

Studies have proven theoretical bolus calculations to be extremely difficult and, when manually calculated, the results were incorrect $63 \%-67 \%$ of the times. ${ }^{3,4}$ Together with the variable eating habits of most children and adolescents, the challenging and time-consuming calculations of insulin doses may encourage patients to take fixed dosages of insulin based on experience and guesses. ${ }^{5}$ Further inappropriate insulin doses confer a possible increased risk of hypoglycemia and greater variability in glucose levels, which is related to cardiovascular risk later in life. ${ }^{6,7}$

An automated bolus calculator (ABC) is a device that calculates the dose of insulin to be administrated based on the following parameters: current blood glucose, estimated amount of carbohydrates to be eaten, insulin-to-carbohydrate ratios (ICR), insulin sensitivity factors (ISF), target blood glucose, and insulin action time. An ABC is a common feature in the software of insulin pumps used in treatment with continuous subcutaneous insulin infusion (CSII). Our study aimed at investigating whether using an $\mathrm{ABC}$ (Accu-Check Aviva Expert, Roche) in MDItreated and carb-counting children and adolescents with T1D would improve not only their metabolic control, but also their quality of life $(\mathrm{QoL})$ and treatment satisfaction.

\section{Methods}

Our study was a 12-month open-labeled crossover trial. Inclusion started in the April 2013 and continued through April 2015. Patients were included from nine study sites in Belgium, Denmark, and Spain.

Eligible patients were between 8 and 18 years of age, had T1D for $>1$ year and had been treated with MDI therapy (fast acting analogue for meals; long acting analogue as basal) for a minimum of 3 months. Finally, their metabolic control upon inclusion had to be suboptimal with $\mathrm{HbA1c}$ between $7.5 \%$ $(57 \mathrm{mmol} / \mathrm{mol})$ and $11 \%(97 \mathrm{mmol} / \mathrm{mol})$.

Patients were all new to the $\mathrm{ABC}$ upon inclusion. Introduction to the $\mathrm{ABC}$ were done in the same manner as for patients starting CSII treatment where an $\mathrm{ABC}$ is a part of the software. Hence, participants and their parents were thoroughly introduced to the device and its functions. Participants were trained in manual bolus calculations using carbohydrate counting, ICR, and ISF. Carbohydrates counting capabilities were evaluated in a meeting with a dietician with T1D experience, ensuring that all participants mastered carbohydrate counting at the level of CSII users. The ABC settings were initially set according to the individual insulin demand and the estimated ISF and ICR before inclusion. During the following visits at the outpatient clinic, these settings were adjusted according to both the participants' experiences and the caregiver's expertise.

Recruited patients agreed to use the $\mathrm{ABC}$ as well as counting carbohydrates and calculating insulin doses on their own during the 12 months study period. Within each study site, participants were randomized (stratified on gender and pubertal stage) to either start with manual carb-counting and the $\mathrm{ABC}$ ( $\mathrm{ABC}$ group) or with simple manual carb-counting and manual bolus calculation (control group). Each period lasted 5 months and they were separated by a 2-month washout period during which data from the patients were not recorded (Fig. 1). At the beginning and at the end of each period participants wore a masked continuous glucose monitor (CGM; DEXCOM G4 Platinum) for a week. HbA1c

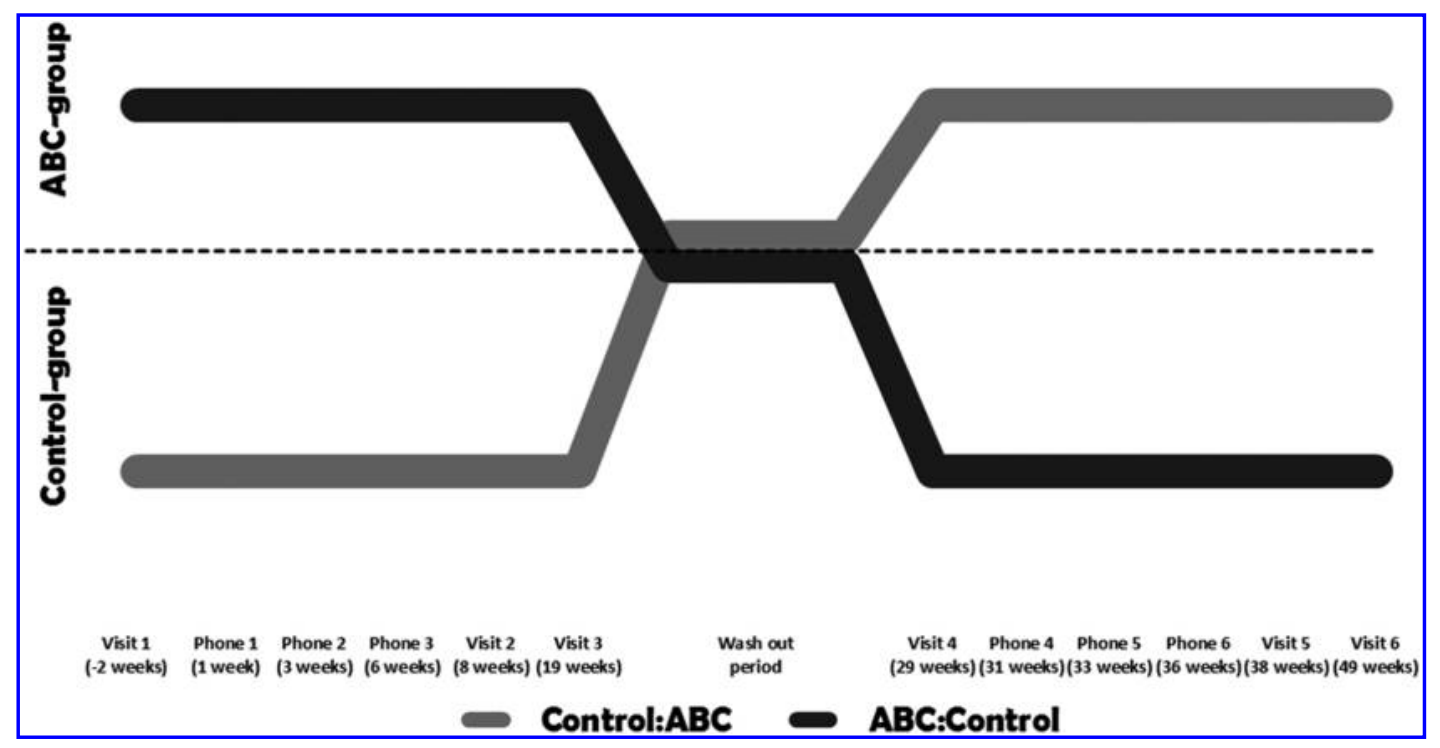

FIG. 1. Visualization of the visits during the trial. "Visits" represents physical visits to the outpatient clinic, whereas "Phone" represents consultations by telephone. ABC, automated bolus calculator. 
was measured three times in each period and consisted of one baseline measurement (visit 1 and 4), one intermediate measurement after 2 months (visits 2 and 5), and one measurement at the end of each period (visits 3 and 6).

All visits and phone calls were conducted by trained staff (pediatric diabetes nurses and doctors) with the facilities and academic knowledge to ensure qualified support and treatment adjustments. Written consent was given from legal guardians (parents of all patients) and ethical approval was obtained for each participation site.

\section{Outcomes}

The main outcome was metabolic control measured by HbA1c. The primary endpoint was the difference in metabolic control at the end of the period (after 5 months). Secondary outcomes included QoL measured by use of Hvidøre questionnaires $^{8}$ and glucose variability monitored for 4-7 days by CGM and described as percentage of time with blood glucose in target (PTARGET $=3.5-10 \mathrm{mmol} / \mathrm{L})$, in high levels $(\mathrm{PHIGH}=>10 \mathrm{mmol} / \mathrm{L})$ or in low levels (PLOW $=$ $<3.5 \mathrm{mmol} / \mathrm{L})$. Furthermore, we looked at the outcomes according to the adherence to $\mathrm{ABC}$ use. If the $\mathrm{ABC}$ was used to calculate $>80 \%$ of boluses, it was considered as high adherence. Use in $<80 \%$ of boluses was considered as low adherence.

\section{Statistical methodology}

A linear model for repeated measurements was used to analyze the HbA1c data. After recommendations for the analysis of crossover data, ${ }^{9}$ the model contains the following effects: visit moment (baseline, 2, 5, 7, 9, and 12 months), sequence ( $\mathrm{ABC}$ :Control vs. Control:ABC), treatment effect after 2 months, treatment effect after 5 months, and finally carryover effects (see Table 1 for a formal presentation of the model). The model parameters were estimated using a mixedeffects model with random effects of patient and center, and with a first-order autoregressive covariance structure for the residuals. The stratification variables gender and pubertal stage (prepubertal vs. pubertal) were added in the model. The primary analysis was also repeated on the subgroup of patients with a minimal adherence of $80 \%$. In an extension of the model, interactions of the treatment parameters with gender and age were added to verify whether the treatment effect depended on both variables. Also, the interaction with the baseline HbA1c was verified fitting the model on visits

Table 1. Parameterization of the Stastistical Model

\begin{tabular}{lll}
\hline Visit & ABC:Control & Control:ABC \\
\hline Baseline & $\pi_{1}$ & $\mathrm{~s}+\pi_{1}$ \\
2 months & $\pi_{2}+\tau_{1}$ & $\mathrm{~s}+\pi_{2}$ \\
5 months & $\pi_{3}+\tau_{2}$ & $\mathrm{~s}+\pi_{3}$ \\
7 months & $\pi_{4}+\vartheta_{1}$ & $\mathrm{~s}+\pi_{4}$ \\
9 months & $\pi_{5}+\vartheta_{2}$ & $\mathrm{~s}+\pi_{5}+\tau_{1}$ \\
12 months & $\pi_{6}+\vartheta_{3}$ & $\mathrm{~s}+\pi_{6}+\tau_{2}$ \\
\hline
\end{tabular}

The $\pi$-parameters refer to the 6 measurement moments. The $\vartheta$-parameters refer to the carry-over effects. The $\tau$-parameters refer to the treatment effect.

$\mathrm{ABC}$, automated bolus calculator.
2-6, with the baseline HbA1c and its interaction with treatment parameters as effects (but omitting the sequence effect, since with data from five visits this is a linear combination of the visit and treatment effects). In addition, the results from an independent $t$-test on visits 2 and 3 were reported. These are between-group analyses because only the data before the washout were considered.

Percentage adherence to the $\mathrm{ABC}$ was analyzed by a linear mixed model with measurement moment (baseline, 2, and 5 months) and sequence (ABC:Control vs. Control:ABC) included as a main effect. Both variables were also included as an interaction effect to test whether the adherence for both sequences changed during the course of the study. Gender and pubertal stage were included as covariates because the randomization was stratified on these variables. Random effects and the covariance structure for the residuals were the same as in the model for HbAlc.

$P$-values $<0.05$ were considered significant. All analyses were performed using $\mathrm{R}$ version 3.3 .3 (2017-03-06) in $\mathrm{R}$ studio.

\section{Sample size calculation}

The sample size calculation was based on a paired $t$-test. This served as an approximation, since the planned analysis referred to a linear model for longitudinal measures. A common standard deviation (SD) of 1 was assumed for the 5months change in HbA1c based on information from a similar study done in adults with $\mathrm{T}_{1} \mathrm{D}^{15}$ and from a retrospective series (unpublished data) in the Leuven center. In case the two 5 month changes are not correlated within each patient (which reflects the worst-case scenario, since the higher the correlation, the lower the needed sample size), the SD for the difference in the 5-months change equals 1.415. Under this scenario, 101 subjects were needed to detect (with $80 \%$ power) a difference of $0.4 \%$ between both groups in 5 months. To account for loss of information due to dropout, 120 patients were intended to be included in the study. The anticipated accrual period was 6 months.

\section{Results}

Since the recruitment tempo proved much slower than anticipated, the recruitment was terminated after inclusion of 65 patients $(57 \%$ boys $[n=37]$ and $43 \%$ girls $[n=28])$. However, as the SD of the 5 months change in HbA1c was lower than assumed in the sample size calculation (1.137 instead of 1.415), it turned out that the actual power of the analysis of the primary outcome was safeguarded. As shown in Table 2, the mean age (SD) for the participants upon inclusion was 13.25 years (2.65) and mean HbA1c (SD) was $8.25 \%$ (1.00) equivalent of $66.7 \mathrm{mmol} / \mathrm{mol}$.

Table 3 shows the results of the linear model used to analyze the changes in HbA1c values. After 5 months with the $\mathrm{ABC}, \mathrm{HbA} 1 \mathrm{c}$ was 0.143 lower $(95 \%$ confidence interval $[\mathrm{CI}]$ : -0.558 to 0.272 ), which was neither clinically relevant (defined as 0.4 in the sample size calculation), nor statistically significant $(P=0.51)$. Also, after 2 months with the ABC, HbA1c was not significantly different $(-0.297,95 \% \mathrm{CI}$ : -0.645 to $0.054 ; P=0.10$ ).

Based on the between-group comparisons before the washout period, the same conclusion was obtained. After 2 months, mean HbA1c equaled $7.98 \pm 0.69(63.7 \mathrm{mmol} / \mathrm{mol})$ 
Table 2. Baseline Characteristics

\begin{tabular}{|c|c|c|c|c|}
\hline Variable & Statistic & Total & Control-ABC & $A B C$-Control \\
\hline \multicolumn{5}{|l|}{ Age } \\
\hline & $N$ & 65 & 32 & 33 \\
\hline & Mean (SD) & $13.25(2.65)$ & $13.09(2.87)$ & $13.39(2.46)$ \\
\hline & Median (IQR) & $13(11-15)$ & $13.5(11-16)$ & $13(11-15)$ \\
\hline & Range & $8-18$ & $8-17$ & $9-18$ \\
\hline \multicolumn{5}{|c|}{ Tanner stage } \\
\hline 1 & $n / N(\%)$ & $15 / 65(23.08)$ & $8 / 32(25)$ & $7 / 33(21.21)$ \\
\hline 2 & $n / N(\%)$ & $5 / 65(7.69)$ & $4 / 32(12.5)$ & $1 / 33(3.03)$ \\
\hline 3 & $n / N(\%)$ & $10 / 65(15.38)$ & $3 / 32(9.38)$ & $7 / 33(21.21)$ \\
\hline 4 & $n / N(\%)$ & $13 / 65(20)$ & $6 / 32(18.75)$ & $7 / 33(21.21)$ \\
\hline 5 & $n / N(\%)$ & $22 / 65(33.85)$ & $11 / 32(34.38)$ & $11 / 33(33.33)$ \\
\hline \multicolumn{5}{|l|}{ BMI } \\
\hline & $N$ & 65 & 32 & 33 \\
\hline & Mean (SD) & $21.26(3.9)$ & $21.33(4.41)$ & $21.2(3.4)$ \\
\hline & Median (IQR) & $21.08(18.45-23.68)$ & $21.05(18.37-23.43)$ & $21.08(18.45-23.68)$ \\
\hline & Range & $14.31-34.12$ & $14.31-34.12$ & $14.44-27.8$ \\
\hline \multicolumn{5}{|l|}{ Gender } \\
\hline Male & $n / N(\%)$ & $37 / 65(56.92)$ & $18 / 32(56.25)$ & $19 / 33(57.58)$ \\
\hline Female & $n / N(\%)$ & $28 / 65(43.08)$ & $14 / 32(43.75)$ & $14 / 33(42.42)$ \\
\hline \multicolumn{5}{|c|}{ Baseline HbA1c (\%) } \\
\hline & $N$ & 65 & 32 & 33 \\
\hline & Mean (SD) & $8.25(1.00)$ & $8.17(1.00)$ & $8.32(1.01)$ \\
\hline & Median (IQR) & $8(7.6-8.7)$ & $7.9(7.57-8.57)$ & $8.1(7.8-8.7)$ \\
\hline & Range & $6.3-12.3$ & $6.3-10.9$ & $6.8-12.3$ \\
\hline
\end{tabular}

$\mathrm{ABC}$, automated bolus calculator; IQR, interquartile range; SD, standard deviation.

in the $\mathrm{ABC}$ :Control group and $8.06 \pm 0.77(64.6 \mathrm{mmol} / \mathrm{mol})$ in the Control:ABC group $(P=0.67)$. After 5 months, mean HbA1c equaled $8.26 \pm 0.94(66.8 \mathrm{mmol} / \mathrm{mol})$ in the $\mathrm{ABC}:-$ Control group and $8.10 \pm 0.98(65.0 \mathrm{mmol} / \mathrm{mol})$ in the Control:ABC group $(P=0.53)$.

The treatment effects did not depend on gender, pubertal stage, or baseline HbA1c. This was tested both after 2 months $(P=0.88, P=0.54$ and $P=0.36)$, and after 5 months $(P=0.25, P=0.33$, and $P=0.74)$.

Table 3. Results from the Mixed Model

\begin{tabular}{lcrlc}
\hline Effect & Parameter & Estimate & \multicolumn{1}{c}{$95 \%$ CI } & P \\
\hline -2 week visit & $\pi_{1}$ & 8.087 & $7.499-8.674$ & $<0.001$ \\
8 week visit & $\pi_{2}$ & 7.996 & $7.36-8.633$ & $<0.001$ \\
19 week visit & $\pi_{3}$ & 8.137 & $7.483-8.792$ & $<0.001$ \\
29 week visit & $\pi_{4}$ & 8.402 & $7.743-9.06$ & $<0.001$ \\
38 week visit & $\pi_{5}$ & 8.380 & $7.552-9.207$ & $<0.001$ \\
49 week visit & $\pi_{6}$ & 8.400 & $7.519-9.28$ & $<0.001$ \\
Treatment & $\tau_{1}$ & -0.297 & -0.648 to 0.054 & 0.104 \\
$\quad$ 2 months & & & & \\
Treatment & $\tau_{2}$ & -0.143 & -0.558 to 0.272 & 0.508 \\
$\quad$ 5 months & & & & \\
Carryover 1 & $\vartheta_{1}$ & -0.552 & -0.99 to -0.113 & 0.016 \\
Carryover 2 & $\vartheta_{2}$ & -0.338 & -1.014 to 0.338 & 0.336 \\
Carryover 3 & $\vartheta_{3}$ & -0.452 & -1.199 to 0.295 & 0.245 \\
Sequence & $\mathrm{s}$ & -0.141 & -0.618 to 0.337 & 0.566 \\
Pubertal & & 0.202 & -0.29 to 0.693 & 0.424 \\
Male & & 0.131 & -0.294 to 0.556 & 0.548 \\
\hline
\end{tabular}

Fixed parameter estimates for a mixed effect model that is used to evaluate the treatment effect of the AVIVA Expert vs the Control condition. A negative estimate for treatment refers to a lower HbAlc under the Aviva Expert use.

$\mathrm{CI}$, confidence interval.
Because the effect of an $\mathrm{ABC}$ is conditioned by consistent use, we proceeded to analyze only participants with high adherence to the ABC. In total $69 \%$ of participants $(n=45)$ had high adherence, whereas the remaining had either low adherence $(n=12)$ or no data recorded regarding adherence $(n=8)$. When analyzing the subgroup with high adherence, there was still no evidence for a treatment effect. The estimates after 2 months $\left(\tau_{1}\right)$ and after 5 months $\left(\tau_{2}\right)$ equaled -0.215 (95\% CI: -0.605 to $0.176 ; P=0.29$ ) and -0.023 (95\% CI: -0.474 to $0.428 ; P=0.92$ ), respectively. Adherence to $\mathrm{ABC}$ was high in $92.6 \%$ and $85.1 \%$ of the visits in the $\mathrm{ABC}$ :Control sequence and the Control:ABC sequence, respectively. The difference in mean adherence was not significant between the sequences $(P=0.10)$.

In addition to $\mathrm{HbAlc}$, we analyzed other metabolic parameters such as the hypoglycemia and ketoacidosis events, average blood glucose, time spent with blood glucose levels within the target range (PTARGET), in high levels (PHIGH), or in low levels (PLOW). No difference between the ABC group and the control group could be demonstrated regarding the average glucose, PHIGH, PTARGET, and PLOW glucose variation (detailed results not shown). The prevalence of hypoglycemic and diabetic ketoacidosis events was too low during the study period to fit a formal model.

Neither participants' QoL, parents' QoL, or the treatment satisfaction improved during the intervention period and no differences were seen between the users of the $\mathrm{ABC}$ and the controls.

\section{Discussion}

Our study found no significant lowering of $\mathrm{HbAlc}$ or blood glucose variation in children and adolescents treated with 
MDI, when adding an $\mathrm{ABC}$ as the Accu-Check Aviva Expert to their treatment. Nor could we find significantly improved QoL or treatment satisfaction in the participants or their parents.

The initial power calculations found that with 65 included subjects, there would only be $61.2 \%$ power (under the assumed SD). However, based on the included subjects, the SD of the difference in changes was much narrower than anticipated. Calculations with the actual SD revealed $79.8 \%$ power to detect a difference of $0.4 \%$ with our 65 participants. So even though inclusion tempo limited the number of participants, the study still had sufficient power to make valid conclusions.

Because of increased insulin resistance ${ }^{10}$ and the balancing between growing independence and the need of assistance and supervision from parents, children and adolescents with T1D can be a particularly difficult group to treat. ${ }^{11}$ As a way to ease up these challenges, the effect of an $\mathrm{ABC}$ have been examined in several studies. ${ }^{12-15}$

Another study investigating the use of the Cozmo Pump Calculator in 40 children and adolescents with T1D treated with CSII but could not demonstrate glucose metabolism closer to the normal range (lower $\mathrm{HbAlc}$ ) in patients using the ABC either. ${ }^{12}$ However, they did find that more blood glucose measurements were within the target range. Their participants had a relatively low starting HbAlc (7.6\%/ $59.6 \mathrm{mmol} / \mathrm{mol}$ ), which was speculated to be one of the reasons they did not see an effect. Indeed, the lower HbAlc value prior of an intervention, the less you might expect $\mathrm{HbA1c}$ to decrease further. Our patients had a mean $\mathrm{HbA1c}$ of $8.25 \%(66.7 \mathrm{mmol} / \mathrm{mol})$ upon inclusion, and still no effect could be demonstrated. This could point to the fact that other factors may be in play for the ABC to be effective. Another study used the same ABC as in our study (Accu-Check Aviva Expert) and investigated the combined effect of carbcounting capabilities and the use of an ABC. They included 85 children treated with MDI and divided them into four groups according to use of $\mathrm{ABC}$ and carb-counting skills. ${ }^{13}$ The two groups using an ABC improved their metabolic control after both 6 and 18 months. These improvements were seen despite both groups started with HbA1c levels $<8 \%$ $(63.9 \mathrm{mmol} / \mathrm{mol})$. Interestingly, only the group that had not been carb-counting before inclusion in the study had significant improvements. This underlines the importance of carb-counting. Hence, it seems, that correct estimation of carbohydrates being the basis for correct insulin dose calculation either manually or automatically has a significant impact on metabolic outcome.

Another important factor in benefitting from the use of a new device is consistent use. We performed a substudy analysis only including participants using the $\mathrm{ABC}$ in $>80 \%$ of boluses. High adherence did not change the outcome of our results. Another study had the same hypothesis and showed that children and adolescents with high-frequency use of an $\mathrm{ABC}$ had significantly better metabolic control compared with low-frequency users. ${ }^{14}$ In this study, high-frequency use was defined as use in just $>50 \%$ of boluses compared with $80 \%$ in our study. Another important difference is the comparison between high- and low-frequency users in their study, whereas we used participants as their own control (crossover), removing potential behavioral differences explaining the adherence and potentially the outcome.

In adults, one of the most recent studies demonstrated a significant improvement in the proportion of MDI-treated patients who obtained near-to-normal metabolic control (HbA1c $<7.5 \% /<58 \mathrm{mmol} / \mathrm{L}$ ) during 4 months of ABC use. However, they could not show any differences in HbAlc between the intervention group and the control group at the end of the study, neither when comparing exact numbers nor when comparing the change from the initial HbA1c. ${ }^{16}$

A randomized controlled pilot study from 2012 examined whether an $\mathrm{ABC}$ had an beneficial effect on the metabolic control in T1D adults with poor metabolic control. ${ }^{15}$ This study found significant improvement in both interventionarms (carb-counting and carb-counting+ABC) in relation to metabolic control, but no difference between the two interventions arms was observed. Only when accounting for the initial HbA1c values, an additive effect could be identified with the use of an ABC. Although the initial difference was not significant, the patients who were in the carb-counting $+\mathrm{ABC}$ arm had a lower initial HbAlc of $8.8 \%(72.7 \mathrm{mmol} / \mathrm{mol})$, whereas the carb-counting arm had an initial HbA1c of $9.2 \%$ $(77.0 \mathrm{mmol} / \mathrm{mol})$. Our patients had an even lower initial HbA1c of $8.25 \%$ (66.7 mmol/mol), which could influence our negative finding on the metabolic outcome. However, our mixed model, including the baseline HbA1c, did not change these findings.

Our intervention period lasted 5 months (19 weeks), which could also influence the outcome. One study found a nonsignificant reduction in HbA1c after 3 months, but after 6 months the reduction was significant. ${ }^{17}$ Certainly, it takes time to know and master the functions of an $\mathrm{ABC}$ and the need for a longer intervention period could be argued. In a $7+7$ days crossover design another study showed that during the 7 day intervention, significantly less correction boluses were taken and significantly less carbohydrates were needed to treat hypoglycemia. ${ }^{5}$ This study did not check for changes in HbA1c, most likely due to the short intervention period. One could argue that a 7 days intervention is not representative of a long-term treatment since it is easier to stay focused for 1 week compared with 5 months. Finally one study had a 12 months intervention period and found that the group using an $\mathrm{ABC}$ had significantly lower $\mathrm{HbA} 1 \mathrm{c}$ already after 3 months and also after 6,9 , and 12 months. ${ }^{18}$ Further the study showed that after 6 and 9 months a significantly higher percentage of participants had HbA1c $<7.5 \%(58.5 \mathrm{mmol} / \mathrm{mol})$ in the intervention arm. ${ }^{18}$ Interestingly participants in the intervention group also showed a significant increase in SMBG measurements, a factor known to improve the glycemic control independently.

The ABACUS study also tested the use of the AccuCheck Aviva Expert (ABC); however, their study period was 26 weeks and the participants were adults ( $>18$ years of age). ${ }^{19}$ In their study, the intervention group reduced $\mathrm{HbA} 1 \mathrm{c}$ more than the control group and more patients in the intervention group reduced their $\mathrm{HbA} 1 \mathrm{c}$ with $>0.5 \%$. Interestingly this study further showed that younger patients used and accepted suggested boluses from the device to a lesser extent than the older participants. This tendency can be one of the major reasons why we could not find any improvement in the metabolic control in our study. Compliance can be a general problem in adolescents and omitted or false blood glucose measurement is not uncommon. ${ }^{20}$ Although participants in studies generally can be thought of as being interested in following the guidelines for the study, even minor omitted boluses can have huge impact. Burdick et al. 
examined the effect of missed meal boluses in teenagers with T1D treated with CSII, and demonstrated that as little as one missed meal bolus per week resulted in significantly higher HbA1c levels. ${ }^{21}$

Omitted boluses can be expected to be just as high in frequency during the intervention period as in the control period. An $\mathrm{ABC}$ does not change this problem and has limited effect if no blood glucose is measured or no carbohydrates are entered. These problems are common in children and adolescents. One study on adolescents showed that $38 \%$ had missed $>15 \%$ of the bolus insulin the day before. ${ }^{22}$ Another study used anonymous questionnaires in 144 adolescents to show that $25 \%$ had missed insulin injections the previous 10 days, $29 \%$ had made up blood glucose results because the real measurement were too high, and finally $29 \%$ had completely made up blood glucose measurements. ${ }^{23}$

When it comes to glucose variability in children using an $\mathrm{ABC}$, the results have been just as conflicting. One study found more blood glucose measurements within target, ${ }^{12}$ whereas another study found increased risk of low blood glucose in inexperienced carb-counters using an $\mathrm{ABC}$. $^{13}$ When participants in the later study continued the use of an $\mathrm{ABC}$, more patients had lower risks of high and low blood glucose after 18 months. ${ }^{13}$ Like us, others could not find any differences in variability. ${ }^{24}$ Measurement of glucose variability is ideally performed by long-term blinded CGM monitoring; however, in a study lasting several months, it can be quite challenging. We could not demonstrate any change in hypoglycemic or ketoacidosis events due to very few events. A similar problem has been seen in other studies. ${ }^{12,13}$

Finally, previous studies have demonstrated an improved treatment satisfaction in patients having an $\mathrm{ABC}^{15,24}$ and improved QOL during the intervention period with the ABC. ${ }^{24}$ In our study, no changes could be seen in QOL or treatment satisfaction between the intervention group and the control group. A large retrospective survey of subjects using an $\mathrm{ABC}$ together with MDI found that $84.9 \%$ of subjects felt that using an $\mathrm{ABC}$ was easier than performing manual bolus calculations, $78.8 \%$ improved their confidence in calculations, and $52 \%$ reduced their fear of hypoglycemia. ${ }^{25}$ These results came from 560 patients with T1D (31\% children and adolescents) and participants had been using an $\mathrm{ABC}$ for 4-12 weeks before answering the questionnaires. Increased confidence and reduced fear of hypoglycemia could be the first step toward improved metabolic control if the intervention went on and were followed up by qualified optimization of the $\mathrm{ABC}$ use. Although causality cannot be determined, associations have showed that adolescents missing $<15 \%$ boluses per day have higher treatment satisfaction, whereas those missing $>15 \%$ boluses have a greater struggle with their daily insulin treatment. ${ }^{22}$

Our study has several strengths. First of all, it is an international multicenter crossover intervention study performed in centers with expertise in treatment of pediatric and adolescent diabetes. The initial mean $\mathrm{HbA1c}$ was $8.2 \%$ $(66.1 \mathrm{mmol} / \mathrm{mol})$. This is high enough to believe that beneficial effects of an $\mathrm{ABC}$ should be revealed and not concealed by fairly well-controlled participants as speculated by Enander et al. ${ }^{12}$ Further the crossover design has earlier been suggested as the next step by Rabbone et al. who found improved glycemic control in patients who were new to carbcounting and were using an $\mathrm{ABC} .{ }^{13}$ Crossover studies take into account the beneficial effects seen in research just because of their participation and not because of the intervention (Hawthorne effect). The intervention/control phases in our study were long (19 weeks) with a 10 weeks washout period in between. This should be sufficient to minimize the carryover effect. In contrast to crossover designs in the field of pharmacokinetics (e.g., 10 times the half-life time) there is no norm for how long the washout period should last in studies examining behavioral effects. Therefore, we introduced parameters (i.e., nuisance parameters) in the formal model to account for these unwanted effects. Being an international multicenter trial could also be a limitation to our study. Multicenter studies are naturally clustered in design that can cause inter-center differences in the treatment routines. Such differences were sought eliminated by careful guidelines in the protocol. Owing to the limited number of participants, subgroup analysis for all nine centers participating would give to small sample sizes to make valid conclusions.

In theory, the beneficial effects of an $\mathrm{ABC}$ together with MDI treatment of T1D children and adolescents are many. However, none could be demonstrated in this study, and previous published results have been conflicting. Some of the major challenges in $\mathrm{ABC}$-assisted treatment guidance are that the full outcome is reliant on correct and consequent usage. ${ }^{4}$ Furthermore, if patients master carb-counting, their metabolic outcome typically leads to a lower $\mathrm{HbAlc}$ regardless of an ABC. ${ }^{26}$ Furthermore, even though no apparent beneficial effect can be seen in the metabolic control, any beneficial effect on QoL or treatment satisfaction would also be of tremendous interest in the treatment of a life-long disease such as T1D.

In conclusion, our study did not demonstrate a clinically relevant change in any tested outcome parameters by adding an $\mathrm{ABC}$ to the MDI treatment of children and adolescents when it comes to metabolic control, glucose variability, or QoL. Omitted insulin boluses and is a possible explanation of the missing effect of an $\mathrm{ABC}$ in children and adolescents with T1D, since exact calculations of boluses is second to a sufficient number of boluses during the day. Further studies on this subject is warranted.

\section{Acknowledgments}

The $\mathrm{ABC}$ consortium thanks Roche International and Roche affiliates in Denmark, Belgium, and Spain for their contributions in providing the eCRF and unrestricted research grants for the completion of the study. Further, the involved diabetes nurse educators played an important role in the study and deserve acknowledgement: Bontinck, Magda; Devisscher, Carine; Goicoechea, Irune; Hertz, Anne Marie; Jørgensen, Lene Kølle; Vandoorne, Eva; and Yoldi, Carmen. The ABC consortium: Casteels, Kristina; Corripio, Raquel; Fieuws, Steffen; González-Vergaz, Amparo; CardonaHernandez, Roque; Johannesen, Jesper; Kristensen, Kurt; Madsen, Jens Otto Broby; Ramon-Krauel, Marta; Sánchez, Jacobo Pérez; Van Aken, Sara; Vanbesien, Jesse; and Vanbrabant, Koen.

\section{Author Disclosure Statement}

No competing financial interests exist. 


\section{References}

1. Effect of intensive diabetes treatment on the development and progression of long-term complications in adolescents with insulin-dependent diabetes mellitus: diabetes Control and Complications Trial. Diabetes Control and Complications Trial Research Group. J Pediatr 1994;125:177-188.

2. Diabetes Control and Complications Trial Research Group, Nathan DM, Genuth S, et al.: The effect of intensive treatment of diabetes on the development and progression of long-term complications in insulin-dependent diabetes mellitus. N Engl J Med 1993;329:977-986.

3. Sussman A, Taylor EJ, Patel M, et al.: Performance of a glucose meter with a built-in automated bolus calculator versus manual bolus calculation in insulin-using subjects. J Diabetes Sci Technol 2012;6:339-344.

4. Glaser NS, Iden SB, Green-Burgeson D, et al.: Benefits of an insulin dosage calculation device for adolescents with type 1 diabetes mellitus. J Pediatr Endocrinol Metab 2004; 17:1641-1651.

5. Gross TM, Kayne D, King A, et al.: A bolus calculator is an effective means of controlling postprandial glycemia in patients on insulin pump therapy. Diabetes Technol Ther 2003;5:365-369.

6. Borg R, Kuenen JC, Carstensen B, et al.: $\operatorname{HbA}_{1}(\mathrm{c})$ and mean blood glucose show stronger associations with cardiovascular disease risk factors than do postprandial glycaemia or glucose variability in persons with diabetes: the A1C-Derived Average Glucose (ADAG) study. Diabetologia 2011;54:69-72.

7. Bragd J, Adamson U, Bäcklund LB, et al.: Can glycaemic variability, as calculated from blood glucose self-monitoring, predict the development of complications in type 1 diabetes over a decade? Diabetes Metab 2008;34(Pt 1):612-616.

8. Skinner TC, Hoey H, McGee HM, et al.: A short form of the Diabetes Quality of Life for Youth questionnaire: exploratory and confirmatory analysis in a sample of 2,077 young people with type 1 diabetes mellitus. Diabetologia 2006;49:621-628.

9. Jones B, Kenward MG: Design and Analysis of Crossover Trials. London: Chapman and Hall, 1989.

10. Amiel SA, Sherwin RS, Simonson DC, et al.: Impaired insulin action in puberty. A contributing factor to poor glycemic control in adolescents with diabetes. $\mathrm{N}$ Engl J Med 1986;315:215-219.

11. Grey M, Davidson M, Boland EA, et al.: Clinical and psychosocial factors associated with achievement of treatment goals in adolescents with diabetes mellitus. J Adolesc Health 2001;28:377-385.

12. Enander R, Gundevall C, Strömgren A, et al.: Carbohydrate counting with a bolus calculator improves post-prandial blood glucose levels in children and adolescents with type 1 diabetes using insulin pumps. Pediatr Diabetes 2012;13: 545-551.

13. Rabbone I, Scaramuzza AE, Ignaccolo MG, et al.: Carbohydrate counting with an automated bolus calculator helps to improve glycaemic control in children with type 1 diabetes using multiple daily injection therapy: an 18-month observational study. Diabetes Res Clin Pract 2014;103: 388-394.

14. Ziegler R, Rees C, Jacobs N, et al.: Frequent use of an automated bolus advisor improves glycemic control in pe- diatric patients treated with insulin pump therapy: results of the Bolus Advisor Benefit Evaluation (BABE) study. Pediatr Diabetes 2016;17:311-318.

15. Schmidt S, Meldgaard M, Serifovski N, et al.: Use of an automated bolus calculator in MDI-treated type 1 diabetes: the BolusCal Study, a randomized controlled pilot study. Diabetes Care 2012;35:984-990.

16. Vallejo-Mora MDR, Carreira-Soler M, Linares-Parrado F, et al.: The Calculating Boluses on Multiple Daily Injections (CBMDI) study: a randomized controlled trial on the effect on metabolic control of adding a bolus calculator to multiple daily injections in people with type 1 diabetes. J Diabetes 2017;9:24-33.

17. Maurizi AR, Lauria A, Maggi D, et al.: A novel insulin unit calculator for the management of type 1 diabetes. Diabetes Technol Ther 2011;13:425-428.

18. Garg SK, Bookout TR, McFann KK, et al:: Improved glycemic control in intensively treated adult subjects with type 1 diabetes using insulin guidance software. Diabetes Technol Ther 2008;10:369-375.

19. Ziegler R, Cavan DA, Cranston I, et al.: Use of an insulin bolus advisor improves glycemic control in multiple daily insulin injection (MDI) therapy patients with suboptimal glycemic control: first results from the ABACUS trial. Diabetes Care 2013;36:3613-3619.

20. Wilson DP, Endres RK: Compliance with blood glucose monitoring in children with type 1 diabetes mellitus. J Pediatr 1986;108:1022-1024.

21. Burdick J, Chase HP, Slover RH, et al.: Missed insulin meal boluses and elevated hemoglobin A1c levels in children receiving insulin pump therapy. Pediatrics 2004; 113(Pt 1):e221-e224.

22. Olinder AL, Kernell A, Smide B: Missed bolus doses: devastating for metabolic control in CSII-treated adolescents with type 1 diabetes. Pediatr Diabetes 2009;10:142-148.

23. Weissberg-Benchell J, Glasgow AM, Tynan WD, et al.: Adolescent diabetes management and mismanagement. Diabetes Care 1995;18:77-82.

24. Gonzalez C, Picón MJ, Tomé M, et al.: Expert study: utility of an automated bolus advisor system in patients with type 1 diabetes treated with multiple daily injections of insulin-a crossover study. Diabetes Technol Ther 2016; 18:282-287.

25. Barnard K, Parkin C, Young A, et al.: Use of an automated bolus calculator reduces fear of hypoglycemia and improves confidence in dosage accuracy in patients with type 1 diabetes mellitus treated with multiple daily insulin injections. J Diabetes Sci Technol 2012;6:144-149.

26. Mehta SN, Quinn N, Volkening LK, et al.: Impact of carbohydrate counting on glycemic control in children with type 1 diabetes. Diabetes Care 2009;32:1014-1016.

Address correspondence to: Jesper Johannesen, MD, DMSc

Department of Pediatrics

Herlev University Hospital Herlev Ringvej 75

Herlev 2730

Denmark

E-mail: jesper.johannesen@regionh.dk 